\title{
Development of a solid bolt/nut model utilized in 3D-FE structural analysis for bolted joints
}

\author{
Masaya HAGIWARA* and Tomohiro KAWAMURA** \\ * Department of Electrical and Mechanical Engineering, Nagoya Institute of Technology \\ Gokiso-cho, Showa-ku, Nagoya, Aichi-pref. 466-8555, Japan \\ E-mail: hagiwara.masaya@nitech.ac.jp \\ ** Graduate School of Nagoya Institute of Technology \\ Gokiso-cho, Showa-ku, Nagoya, Aichi-pref. 466-8555, Japan
}

Received: 3 July 2017; Revised: 28 August 2017; Accepted: 11 December 2017

\begin{abstract}
This study aims to develop a solid bolt/nut model utilized in 3D-FE analysis for the strength design of mechanical structures with bolted joints to obtain the internal forces and the bending moment acting on the bolt/nut assembly. A solid bolt/nut model has an advantage to save the time for calculation drastically since it requires fewer numbers of elements and contact surfaces. On the other hand, the solid model has to have the same axial and flexural stiffnesses as the actual bolt/nut assembly to maintain the accuracy of the equilibrium of forces and displacement in the bolted joint and of the internal forces or stresses acting on the bolt/nut assembly calculated. This study depicts how to determine the design parameters for the solid model directly from the specifications of actual bolt/nut assemblies appropriately. To demonstrate the validity of the solid model developed, the stresses acting on the bolt/nut assembly in a cylinder cover bolted joint subjected to internal pressure are calculated by 3D-FE analysis using (a) fully modeled axi-symmetric bolt/nut assembly and (b) the solid bolt/nut model developed. The results obtained using the solid model proposed coincide accurately with the ones using the fully modeled axi-symmetric bolt/nut assembly model, and show good agreement with the measurement using the strain gauges put on the bolt shank as well. This clearly highlights the effectiveness and usefulness of the solid model developed.
\end{abstract}

Keywords : Machine element, Bolted joint, Strength design, 3D-FE analysis, Bolt/nut assembly, Solid model, Equivalent stiffness

\section{Introduction}

In the strength design for bolted joints in a mechanical structure, it is essential to obtain the internal forces and the bending moment acting on the bolt/nut assembly concerned when the external load is applied. Since bolted joints are very complex statically indeterminate structures, the equilibrium of deformation on the bearing surfaces of bolt and nut is necessary for the analysis. Therefore, many studies have been conducted on the stiffness of bolt/nut assembly and of the clamped members (for example, Bickford, 1995) mostly by using so called "elastic theory".

Recent development of computer technology enables to utilize 3D-FE analysis for bolted joint design. In VDI 2230 Blatt2 (2014), four categories (Model Classes I to IV) of 3D-FE analysis are defined and characterized in relation to the design stages of bolted joints. In Model Class IV, fully modeled bolt/nut assembly is installed to obtain the internal forces and the local stresses acting on the bolt/nut assembly precisely. In such a case, the stiffness can be maintained automatically just by setting the material properties such as Young's modulus and Poisson's ratio. However, the use of Model Class IV is not recommended at the early design stage in which trial-and-error process is normally required due to its long preparation time for modeling, and its long processing time to cope with the complex contact problems and with the increasing number of elements. It should be used at the final evaluation stage of the design. For the purpose of determining the internal forces and the bending moment acting on the bolt/nut assembly, Model Class III is suitable. 
Model Class III is 3D FE model in which fully modeled bolt/nut assembly is replaced by "equivalent volume body without thread" having the same compliances (or stiffnesses). It gives the same results of internal forces as Model Class IV for any types of bolted joints provided that the equivalent volume body (solid model) has the same stiffnesses as the practical ones. However, only a method for obtaining the stiffnesses using the classical elastic theory is shown in VDI 2230 Blatt 1 and the experimental determination or adjustment is recommended.

In this study, the bolt/nut model utilized in Model Class III without any limitations is the target to be developed. To realize this, the solid bolt/nut mode has to have both the same axial and flexural stiffnesses as the actual bolt/nut assembly since most of the bolt/nut assemblies in practical bolted joints are subjected to bending (bending moment and/or transverse force) as well as axial tension even though the external load acting on the bolted joint is purely axial tensile force (Galwelat and Beitz, 1981, Bickford, 1995). Such solid models having equivalent stiffness have already been utilized in 3D-FE analysis for specific purposes (for example, Fukuoka et al., 2014). However, the formulation or the generalization of the modeling method in relation to the specifications of the actual bolt/nut assembly has not fully been discussed yet.

The modeling process consists of three steps, (1) to make an axi-symmetric model of bolt/nut assembly, (2) to make an equivalent cylindrical body for free threaded portion, and (3) to make an equivalent solid body for mating threads of bolt/nut assembly. The design parameters of the solid model should be determined directly and only from the specifications of the practical bolt/nut assembly to be modeled in a formulated manner to guarantee the applicability to any types of bolt and nut and their combinations. To demonstrate the validity of the solid model developed, the stresses or the internal forces and the bending moment on the bolt/nut assembly in a cylinder cover bolted joint subjected to internal pressure are calculated using the fully modeled axi-symmetric bolt/nut model and the solid bolt/nut model, respectively, and are compared with the measurement by the strain gauges put on the bolt shank and with the conventional solid model without any features for the mating threads as a reference.

\section{Modeling of bolt/nut assemblies}

\subsection{Axi-symmetric model for bolt/nut assembly}

Figure 1 (b) shows the axi-symmetric model corresponding to the 3D-bolt/nut assembly model shown in Fig.1 (a). Axi-symmetric models are commonly used to obtain the local stress in relation to the fatigue design of bolt/nut assembly subjected to axial tensile force (Furukawa et. al., 2012). Experience shows that the hexagon profile of the head and the nut can be modeled into cylindrical shape having the same volume (diameter of 1.05s) (Fukuoka et al., 2014), and that the effect of the lead angle $\varphi$ of the thread on the deformation (dilation) of the nut is negligibly small (Hagiwara and Ikezawa, 2015).

Figure 3 shows the stiffnesses (spring rates) in axial and transverse directions for free-threaded portions obtained from the models shown in Fig. 2. The calculations were performed for coarse pitch threads from M3 to M12, which have bigger lead angle $(\varphi)$ than fine pitch threads for the same nominal diameter. The results clearly show that the effect of the thread lead (angle $\varphi$ ) on the stiffness is negligibly small.

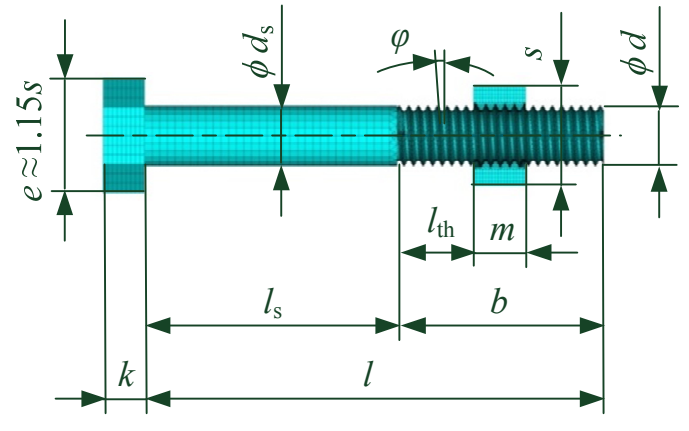

(a) 3D-bolt/nut assembly model

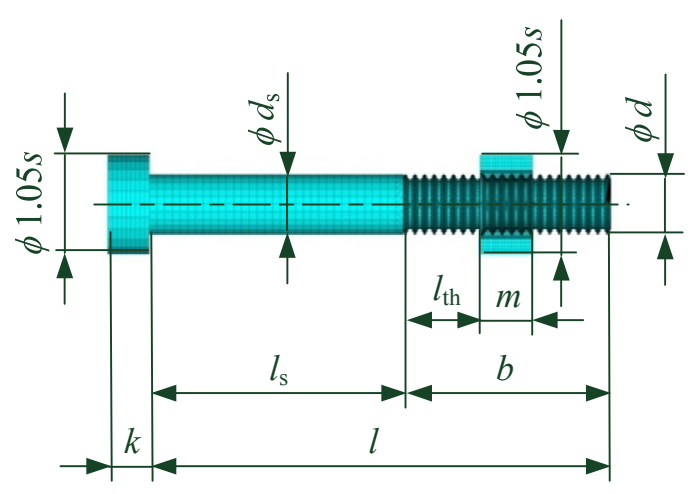

(b) Axi-symmetric bolt/nut assembly model

Fig. 1 Axi-symmetric model for bolt/nut assembly. By using axi-symmeric model (b) instead of 3D-bolt/nut assembly model with lead (a), we can exclude the difficulty and save the time in meshing, i.e., making nodes and elements especially for incomplete threads at the bolt shank and at both ends of the nut (the first and the last turns). However, the total numbers of elements are almost the same. 


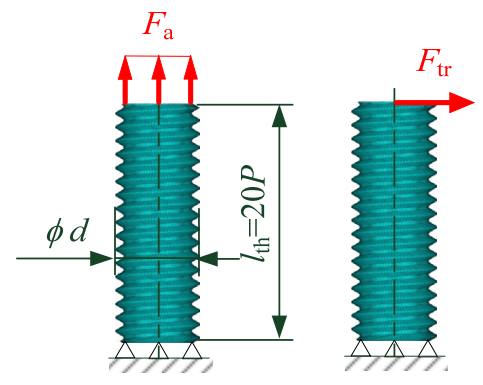

(a) with lead

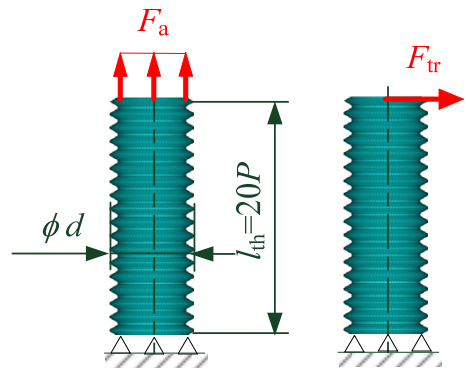

(b) without lead

Fig. 2 The models to compare the stiffnesses in axial and transverse directions for free-threaded portion. Both threads have the same profile with the mean dimensions of the thread tolerance class $6 \mathrm{~g}$. The stiffnesses $K_{\mathrm{a}}$ and $K_{\text {tr }}$ are defined as the elongation per unit axial force, and the deflection per unit transverse force, respectively.

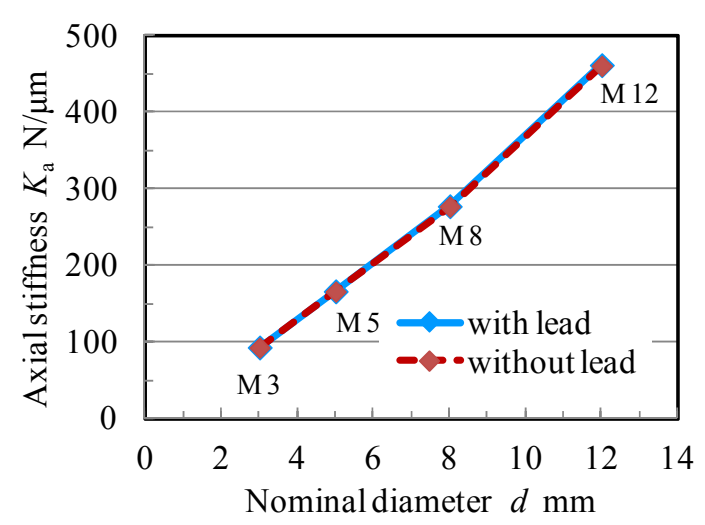

(a) Axial stiffness

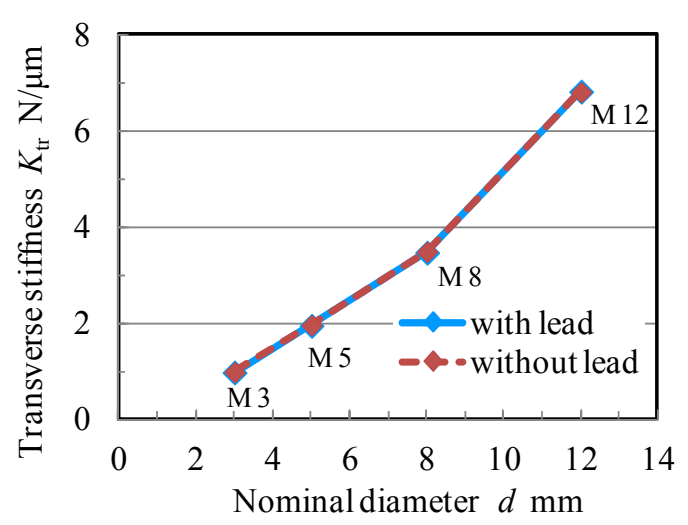

(b) Transverse stiffness

Fig. 3 Comparison of the stiffnesses in axial and transverse directions between 3D-FE model (with lead) and axi-symmetric FE model (without lead) shown in Fig. 2. Young's modulus $E=205.8 \mathrm{GPa}$ and Poisson's ratio $v$ $=0.30$ are assumed. The results clearly show that the effect of the lead angle $\varphi$ of the thread on the stiffness or the deformation of free-threaded portion is negligibly small.

The axi-symmetric model as shown in Fig. 1 (b) is much easier to make, and it helps to decrease the preparation time for making 3D-FE models for bolted joints. However, the number of elements required to make a thread profile could not be changed drastically. Therefore, a saving of the processing time or the calculation time is not expected.

\subsection{Cylindrical model for free threaded portion}

As the first step to decrease the numbers of elements in bolt/nut assembly model, the free-threaded portion is modeled to the cylindrical body having the equivalent diameter of $d_{\text {eq }}$.

Tables 1 and 2 show the equivalent diameter $d_{\mathrm{eq}}$ for free-threaded portion obtained from the results shown in Fig. 3 using the following relationships, respectively:

$$
\begin{aligned}
& K_{\mathrm{a}}=\frac{A_{\mathrm{eq}} \cdot E}{l_{\mathrm{th}}}=\frac{\pi \cdot d_{\mathrm{eq}}^{2} \cdot E}{4 \cdot l_{\mathrm{th}}} \\
& K_{\mathrm{tr}}=\frac{3 \cdot I_{\mathrm{eq}} \cdot E}{l_{\mathrm{th}}^{3}}=\frac{3 \cdot \pi \cdot d_{\mathrm{eq}}^{4} \cdot E}{64 \cdot l_{\mathrm{th}}^{3}}
\end{aligned}
$$

where $E(=205.8 \mathrm{GPa})$ is Young's modulus of the bolt material. The minor diameters $d_{1 \mathrm{~m}}$ and $d_{3 \mathrm{nom}}$, and the diameter $d_{A \mathrm{~s}}$ for the stress area $A_{\mathrm{s} \text { nom }}$ are also shown to determine the equivalent diameter of the solid model in relation to the thread profile, see Fig. 4. 
Table 1 Equivalent diameter $d_{\mathrm{eq}}$ for free-threaded portion for elongation obtained using Eq. (1) in comparison with the minor diameters $d_{1 \mathrm{~m}}$ and $d_{3 \mathrm{nom}}$, and the diameter $d_{\mathrm{As}}$ for the stress area $A_{\mathrm{s} \mathrm{nom}}$.

\begin{tabular}{|c|c|c|c|c|c|c|c|c|c|c|}
\hline \multirow{2}{*}{$\begin{array}{c}\text { Thread } \\
d\end{array}$} & \multirow{2}{*}{$\begin{array}{l}\text { Pitch } \\
P \mathrm{~mm}\end{array}$} & \multirow{2}{*}{$\begin{array}{l}\text { Lead } \\
L \mathrm{~mm}\end{array}$} & \multicolumn{2}{|c|}{ Equiv. diameter } & \multicolumn{4}{|c|}{ Minor diameters } & \multicolumn{2}{|c|}{ Diameter for $A_{\mathrm{s} \text { nom }}$} \\
\hline & & & $d_{\mathrm{eq}} \mathrm{mm}$ & Error \% & $d_{1 \mathrm{~m}}^{\mathrm{b}} \mathrm{mm}$ & Error \% & $d_{3 \mathrm{nom}} \mathrm{mm}$ & Error \% & $d_{A \mathrm{~s}}{ }^{\mathrm{c}} \mathrm{mm}$ & Error \% \\
\hline M3 & 0.5 & $\begin{array}{c}0.5 \\
0^{\mathrm{a}} \\
\end{array}$ & $\begin{array}{l}2.405 \\
2.403\end{array}$ & $\begin{array}{c}- \\
-0.10 \\
\end{array}$ & 2.402 & $\begin{array}{l}-0.16 \\
-0.07 \\
\end{array}$ & 2.387 & $\begin{array}{l}-0.77 \\
-0.68 \\
\end{array}$ & 2.531 & $\begin{array}{l}+5.22 \\
+5.32 \\
\end{array}$ \\
\hline M5 & 0.8 & $\begin{array}{c}0.8 \\
0^{\mathrm{a}}\end{array}$ & $\begin{array}{l}4.067 \\
4.064\end{array}$ & $\begin{array}{c}- \\
-0.07\end{array}$ & 4.063 & $\begin{array}{l}-0.11 \\
-0.04\end{array}$ & 4.019 & $\begin{array}{l}-1.19 \\
-1.12\end{array}$ & 4.252 & $\begin{array}{l}+4.55 \\
+4.62\end{array}$ \\
\hline M8 & 1.25 & $\begin{array}{c}1.25 \\
0^{\mathrm{a}} \\
\end{array}$ & $\begin{array}{l}6.566 \\
6.560 \\
\end{array}$ & $\begin{array}{c}- \\
-0.09 \\
\end{array}$ & 6.560 & $\begin{array}{l}-0.09 \\
-0.00\end{array}$ & 6.467 & $\begin{array}{l}-1.52 \\
-1.42\end{array}$ & 6.826 & $\begin{array}{l}+3.96 \\
+4.06\end{array}$ \\
\hline M12 & 1.75 & $\begin{array}{c}1.75 \\
0^{\mathrm{a}}\end{array}$ & $\begin{array}{l}10.022 \\
10.011\end{array}$ & $\begin{array}{c}- \\
-0.11\end{array}$ & 9.997 & $\begin{array}{l}-0.25 \\
-0.14\end{array}$ & 9.853 & $\begin{array}{l}-1.68 \\
-1.58\end{array}$ & 10.360 & $\begin{array}{r}+3.37 \\
+3.48 \\
\end{array}$ \\
\hline
\end{tabular}

a: Axi-symmetric threads without lead [Fig. 2 (b)].

b: Mean value of $d_{1}$ for thread tolerance class $6 \mathrm{~g} \quad$ (=actual $d_{1}$ value of the model in Fig. 2).

c: $=\left(d_{2 \text { nom }}+d_{3 \text { nom }}\right) / 2$

Table 2 Equivalent diameter $d_{\mathrm{eq}}$ for free-threaded portion for deflection obtained using Eq. (2) in comparison with the minor diameters $d_{1 \mathrm{~m}}$ and $d_{3 \mathrm{nom}}$, and the diameter $d_{\mathrm{As}}$ for the stress area $A_{\mathrm{s} \text { nom }}$.

\begin{tabular}{|c|c|c|c|c|c|c|c|c|c|c|}
\hline \multirow{2}{*}{$\begin{array}{c}\text { Thread } \\
\quad d\end{array}$} & \multirow{2}{*}{$\begin{array}{l}\text { Pitch } \\
P \mathrm{~mm}\end{array}$} & \multirow{2}{*}{$\begin{array}{l}\text { Lead } \\
L \mathrm{~mm}\end{array}$} & \multicolumn{2}{|c|}{ Equiv. diameter } & \multicolumn{4}{|c|}{ Minor diameters } & \multicolumn{2}{|c|}{ Diameter for $A_{\mathrm{s} \text { nom }}$} \\
\hline & & & $d_{\mathrm{eq}} \mathrm{mm}$ & Error \% & $d_{1 \mathrm{~m}}{ }^{\mathrm{b}} \mathrm{mm}$ & Error \% & $d_{3 \text { nom }} \mathrm{mm}$ & Error $\%$ & $d_{A \mathrm{~s}}{ }^{\mathrm{c}} \mathrm{mm}$ & Error \% \\
\hline \multirow{2}{*}{ M3 } & \multirow{2}{*}{0.5} & 0.5 & 2.389 & - & \multirow{2}{*}{2.402} & +0.51 & \multirow{2}{*}{2.387} & -0.11 & \multirow{2}{*}{2.531} & +5.93 \\
\hline & & $0^{\mathrm{a}}$ & 2.394 & +0.21 & & +0.30 & & -0.31 & & +5.71 \\
\hline \multirow{2}{*}{ M5 } & \multirow{2}{*}{0.8} & 0.8 & 4.036 & - & \multirow{2}{*}{4.063} & +0.66 & \multirow{2}{*}{4.019} & -0.43 & \multirow{2}{*}{4.252} & +5.36 \\
\hline & & $0^{\mathrm{a}}$ & 4.042 & +0.15 & & +0.51 & & -0.58 & & +5.20 \\
\hline \multirow{2}{*}{ M8 } & \multirow{2}{*}{1.25} & 1.25 & 6.512 & - & \multirow{2}{*}{6.560} & +0.74 & \multirow{2}{*}{6.467} & -0.70 & \multirow{2}{*}{6.826} & +4.82 \\
\hline & & $0^{\mathrm{a}}$ & 6.521 & +0.13 & & +0.61 & & -0.83 & & +4.69 \\
\hline \multirow{2}{*}{ M12 } & \multirow{2}{*}{1.75} & 1.75 & 9.921 & - & \multirow{2}{*}{9.997} & +0.76 & \multirow{2}{*}{9.853} & -0.68 & \multirow{2}{*}{10.360} & +4.42 \\
\hline & & $0^{\mathrm{a}}$ & 9.917 & -0.04 & & +0.81 & & -0.64 & & +4.47 \\
\hline
\end{tabular}

: Axi-symmetric threads without lead [Fig. 2 (b)].

: Mean value of $d_{1}$ for thread tolerance class $6 \mathrm{~g}$ (=actual $d_{1}$ value of the model in Fig. 2).

c: $=\left(d_{2 \text { nom }}+d_{3 \text { nom }}\right) / 2$

The equivalent diameter $d_{\text {eq }}$ for both elongation and deflection coincides with the mean value of the root diameter $d_{1 \mathrm{~m}}=\left(d_{1 \max }+d_{1 \mathrm{~min}}\right) / 2$, which corresponds to the actual $d_{1}$ value for the model shown in Fig. 2 within $\pm 1 \%$ accuracy. The results also suggest that the root diameter $d_{3}$ used to calculate the axial and flexural compliances of bolt/nut assembly in VDI 2230 Blatt 1 (2014) seems to be a little too small even if the nominal value $\left(d_{3 \mathrm{nom}}=d_{1 \mathrm{nom}}-H / 6\right.$, see Fig. 4) is applied. On the other hand, the diameter of nominal stress area $d_{\mathrm{As}}\left[=\left(d_{2}+d_{3}\right) / 2\right]$ is too large, and should not be used for structural analysis of bolted joints even though it is a good index for obtaining the nominal stress comparable to the strength of the material (Okubayashi et al., 2006).

\subsection{Solid model for mating threads}

In the bolt/nut assembly subjected to tensile force, the radial deformation of the nut called "nut dilation" occurs due to the slippage between the mating threads in addition to the axial deformation due to thread bending. For the axial stiffness of mating threads, the axial deformation is decisive. However, the magnitude of the nut dilation affects the amount of the load shared by each thread mated (Hagiwara et al., 2001), and it may not only change the total axial stiffness of mating threads but also the equilibrium of forces on the bearing surface of the nut in a bolted joint. Therefore, not only the axial deformation but also the radial deformation of the solid model for nuts had better be as close as possible to the actual bolt/nut assembly under tensile loading even though the effect of the coefficient of friction $\mu_{\text {th }}$ between threads which affects the magnitude of the nut dilation cannot be taken into account in solid models.

Figure 5 shows axi-symmetric bolt/nut model and its solid models subjected to axial tensile force. Model A has two 


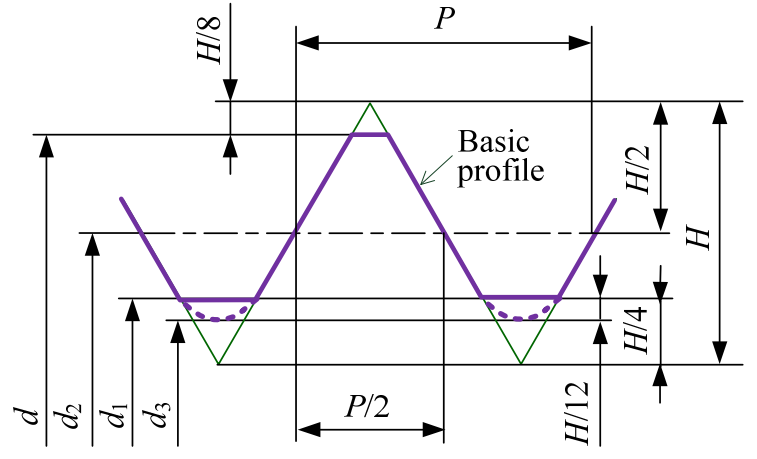

(a) Basic profile (nominal value)

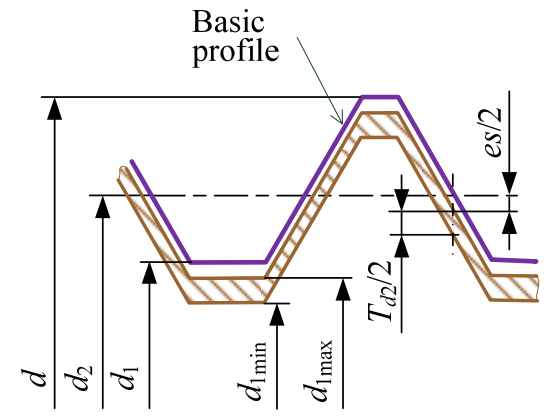

(b) Tolerance positions e, f and $g$

Fig. 4 Basic dimensions and the tolerance zone for external threads with tolerance position(s) (e, f and) g. The limits of size for $d_{1}$ are not specified in ISO 965-1 (1998). However, they can be defined and calculated by using the fundamental deviation es and the tolerance $T_{d 2}$ for pitch diameter $d_{2}$.

circular slits and reduced nut height determined by three parameters $C_{1}$ to $C_{3}$ to control the deformation of nut and the axial and the flexural stiffnesses. On the other hand, Model B has the same geometry (contour) as the practical nut, and multiple conical cavities with the angle $\theta$ and the width $w$ to simulate the behavior of mating threads. Considering the feature that the mating threads can be modeled independently, Model B has a benefit to formulate the method of modeling for various types of nuts.

Figure 6 shows the effect of the individual design parameters on the displacements of the bearing surface of nut in axial and radial directions among three models for M8 bolt and nut with the coefficients of friction $\mu_{\mathrm{th}}$ and $\mu_{\mathrm{b}}$ of 0.15 . The differences of the magnitude of the deformation between bolt/nut assembly (red dashed line) and the conventional model [Model A without slits $\left(C_{1}=0, C_{2}=0\right.$ and $\left.C_{3}=1\right)$ ] suggest the necessity of the modeling for the mating threads to reduce the stiffness. Comparing the results for Model A and Model B, Model B has the advantage that less number of parameters and more linear relationship make the determination of parameters much easier. Furthermore for Model A, the radial displacement becomes considerably smaller and/or the axial displacement becomes considerably larger than

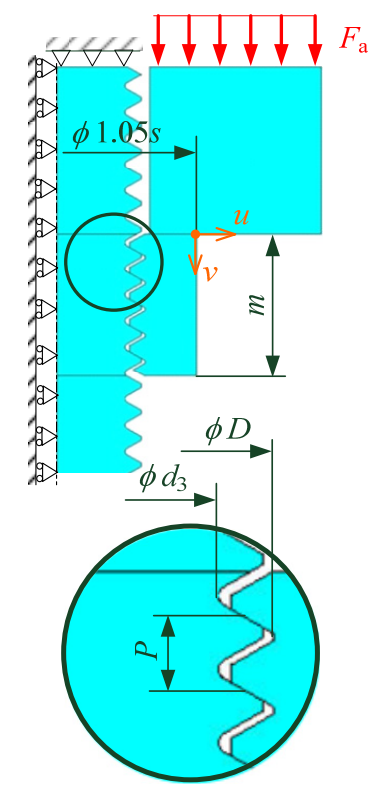

Axi-symmetric bolt/nut assembly

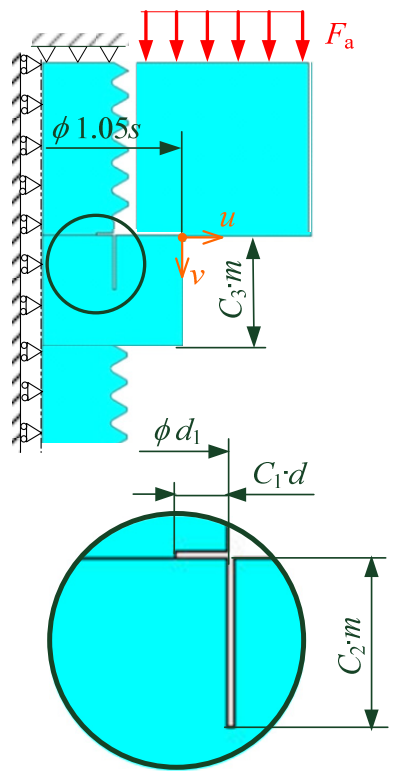

Model A (with slits)

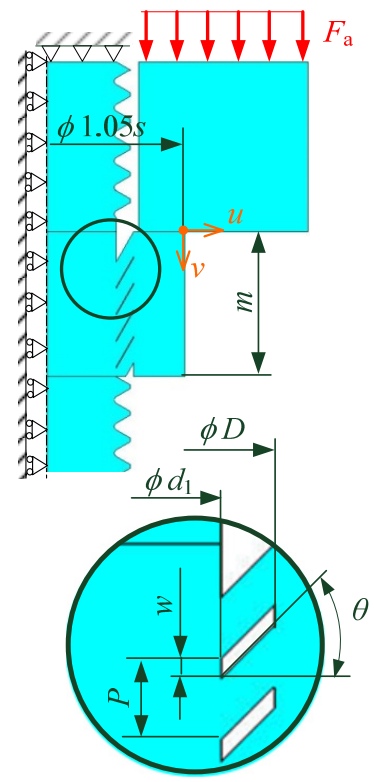

Model B (with cavities)

Fig. 5 Two types of solid models for realizing the similar behavior of mating threads in bolt/nut assembly. Model A has reduced nut height and two circular slits to control the axial and flexural stiffnesses, respectively. On the other hand, in Model B, mating threads are modeled into multiple conical cavities with pitch $P$ taking the conical angle $\theta$ and the width $w$ of cavities as parameters. 

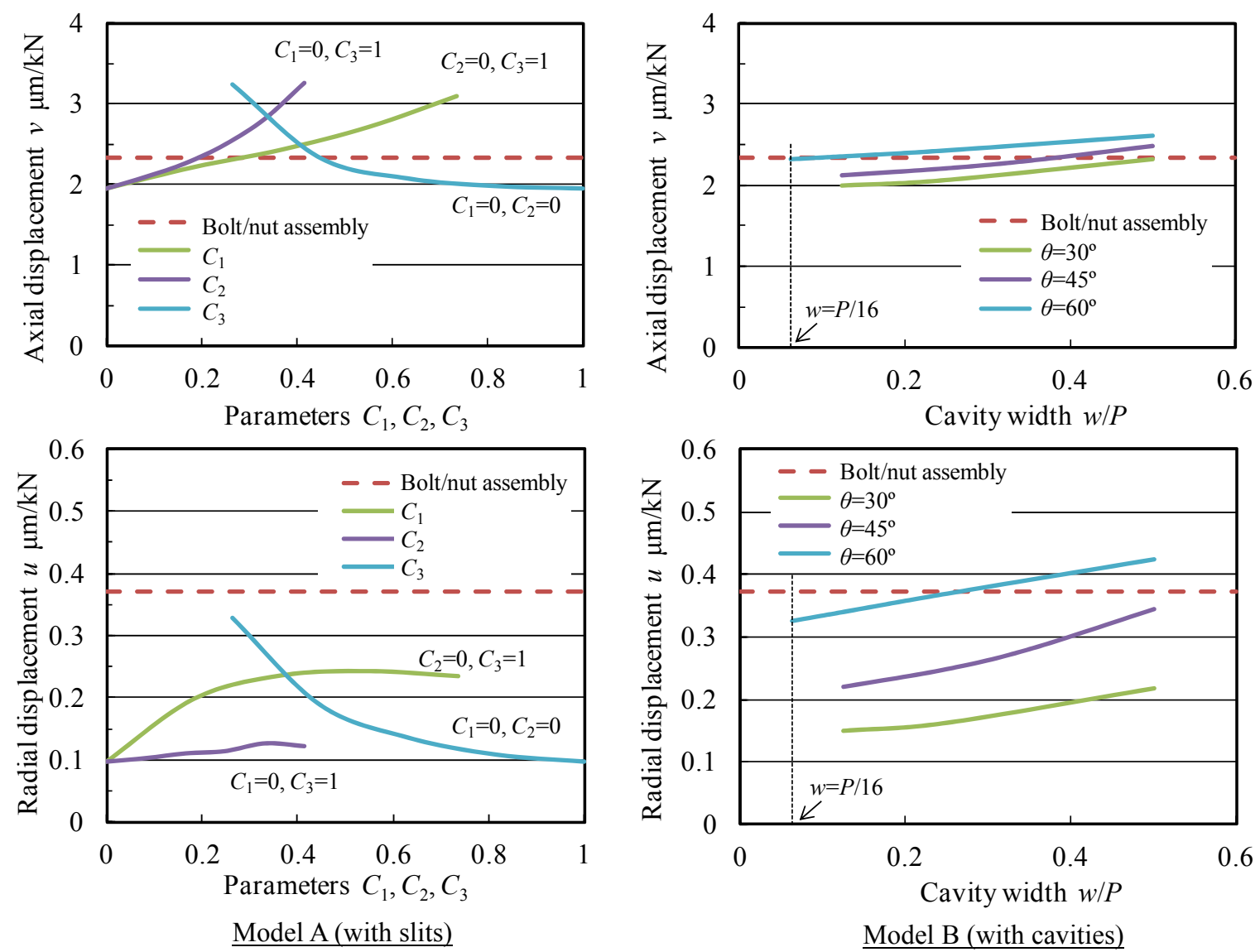

Fig. 6 Effect of the parameters for each solid model shown in Fig. 5 on the deformation of nut in axial and radial directions. For making the appropriate solid models, the parameters have to be selected to realize the similar nut deformation both in axial and radial directions simultaneously. For Model A, it is difficult since the effect of the slits is not sufficient to simulate the radial deformation. From the results for Model B, the cavity angle $\theta$ $=60^{\circ}$ can be selected first since both the deformations $u$ and $v$ are closest to the bolt/nut assembly. The vertical lines show the width $w=P / 16$, which is finally selected as the appropriate value for $\theta=60^{\circ}$. It gives the same stiffnesses as bolt/nut assembly although the radial displacement is a little bit smaller, see Fig. 8 .

bolt/nut assembly even if the optimal set of three parameters can be determined (in this case, $-23 \%$ radial displacement and $+8 \%$ axial displacement for the optimal set of parameters, $C_{1}=0.0825, C_{2}=0.25$ and $C_{3}=0.85$ ). Therefore, Model B was adopted as the solid model for the mating threads of the bolt/nut assembly proposed in this study.

Figure 7 shows the final model of the mating threads to check the axial and flexural stiffnesses. The results are shown in Fig. 8. Both the axial and flexural stiffnesses of the solid model with conical cavities (Model B) with $\theta=60^{\circ}$ and $w=P / 16$ are very similar to those of the axi-symmetric bolt/nut assembly for all the range of nominal designations from M3 to M12 although the radial displacement shown in Fig. 6 is a little bit smaller compared with the axi-symmetric bolt/nut assembly.

Combining the results shown in Tables 1 and 2, and Fig. 8, we can make the solid model directly from the actual geometries and dimensions including the thread profile of the bolt/nut assembly to be modeled as follows:

- Hexagon profile of nut and bolt head: to cylindrical body with outer diameter $1.05 \mathrm{~s}$ and the same height.

- Free-threaded portion: to cylindrical body with diameter $d_{1}$.

- Mating threads: to multiple cavities with pitch $P$, inner diameter $d_{1}$, outer diameter $D$, the angle $\theta=60^{\circ}$ and the width $w=P / 16$.

\section{Verification of the applicability of the solid model for bolt/nut assembly}

Figure 9 shows a cylinder cover bolted joint fastened by eight M8 bolts and subjected to internal pressure $p$ as an 


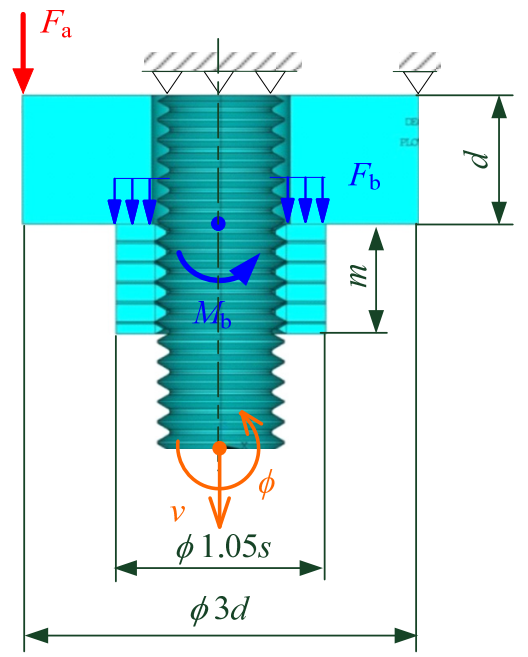

For axi-symmetric bolt/nut assembly

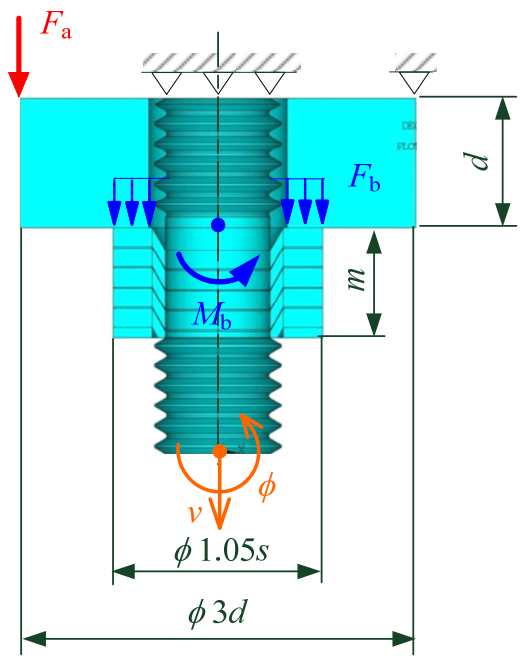

For Model B

Fig. 7 Bolted joint model to check the axial and flexural stiffnesses of the solid model (Model B in Fig. 5) for mating threads. The cavity angle $\theta=60^{\circ}$ and the width $w=P / 16$ are selected as appropriate parameters. The axial and flexural stiffnesses are obtained by using the axial force $F_{\mathrm{b}}$ and the axial displacement $v$, and the bending moment $M_{\mathrm{b}}$ and the slope $\phi$ at the bolt end, respectively.

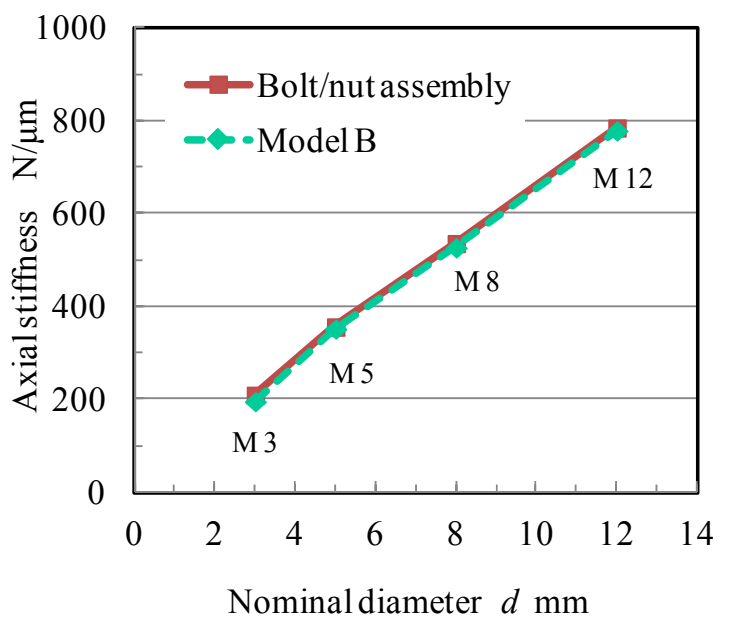

(a) Axial stiffness

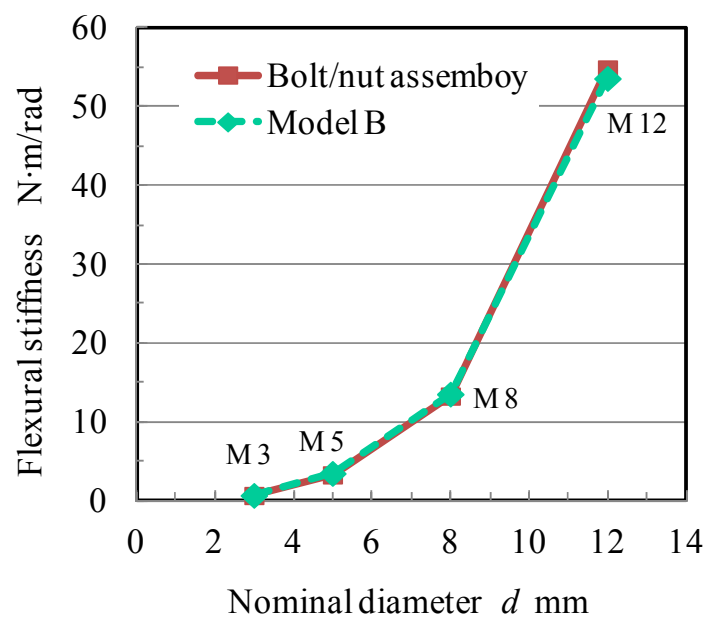

(b) Flexural stiffness

Fig. 8 Comparison of axial and flexural stiffnesses between axi-symmetric bolt/nut assembly and the solid model (Model B with $\theta=60^{\circ}$ and $w=P / 16$ ). Both the axial and flexural stiffnesses of the solid model coincide perfectly with those of the bolt/nut assembly model. It has been confirmed that the nonlinearity or the force dependency on the stiffnesses of bolt/nut assembly is negligibly small through the practical ranges of the external force $F$ or the force $F_{\mathrm{b}}$ and the bending moment $M_{\mathrm{b}}$.

example of multi-bolted joints typically used (Hagiwara et al., 1985). The single-bolted joint model corresponding to Fig. 9 for 3D-FE analysis is shown in Fig. 10, where two types of bolt/nut assembly models, (a) fully modeled axi-symmetric bolt/nut assembly model and (b) solid model (Model B in Fig. 5 with $\theta=60^{\circ}$ and $w=P / 16$, and $d_{\text {eq }}=d_{1}$ ) proposed in this study, are installed. In the FE analysis, the clamp force applied to the joint can be generated by introducing the "offset" of the bolt length against the grip length. The specifications of the clamped members, e.g., the axial compressive stiffness and the geometries such as the eccentricity of the bolt axes, as well as those of bolt/nut assembly affect the magnitude of the clamp force induced by a specific offset value. However, the appropriate offset value can easily be interpolated from the results of the preliminary FE analysis of the joint since there is approximately linear relationship between clamp force and offset value for almost all the cases.

Figure 11 shows the relationship between the external load (axial force per a bolt $W$ ) and the stress acting on the 


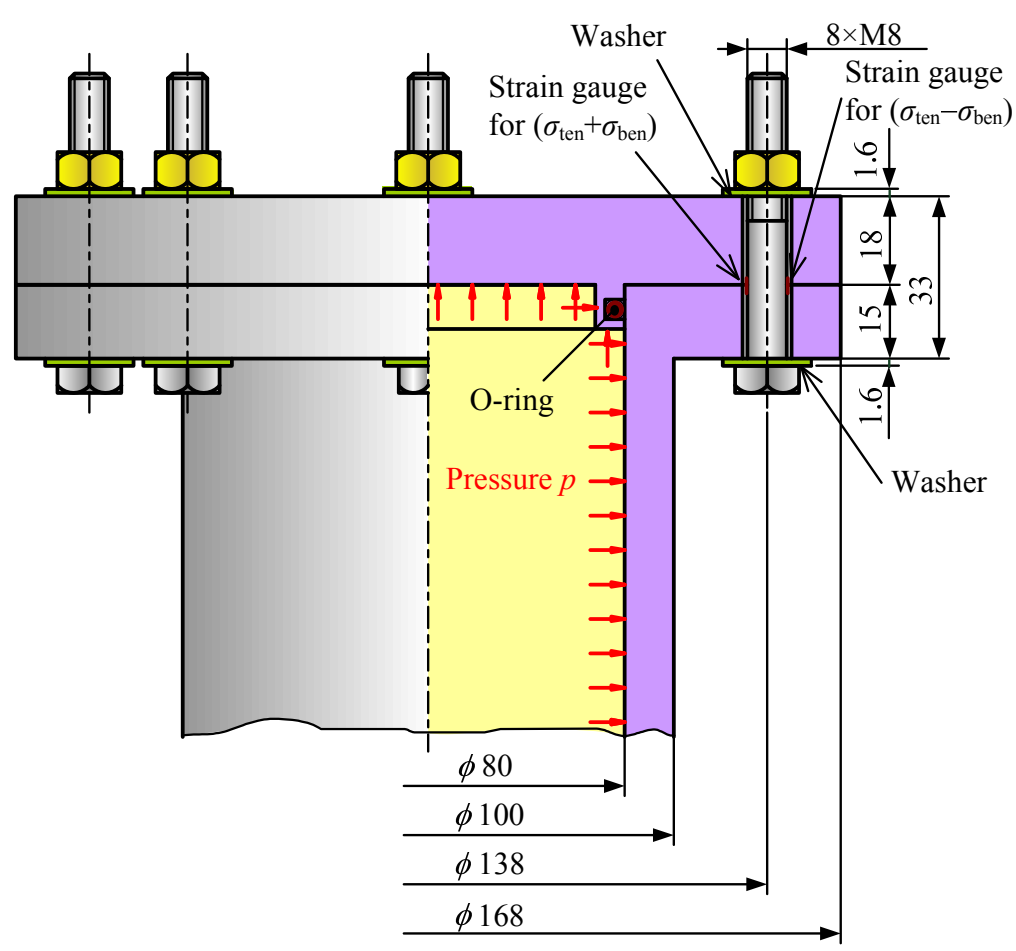

Fig. 9 A cylinder cover bolted joints as an example of typical multi-bolted joints. The cylinder cover is fastened by eight M 8 bolts and nuts with washers, and subjected to internal pressure $p$ of 0 to $14 \mathrm{MPa}$. The tensile and bending stresses $\left(\sigma_{\text {ten }} \pm \sigma_{\text {ben }}\right)$ acting on the bolt are measured by strain gauges put on the bolt shank.

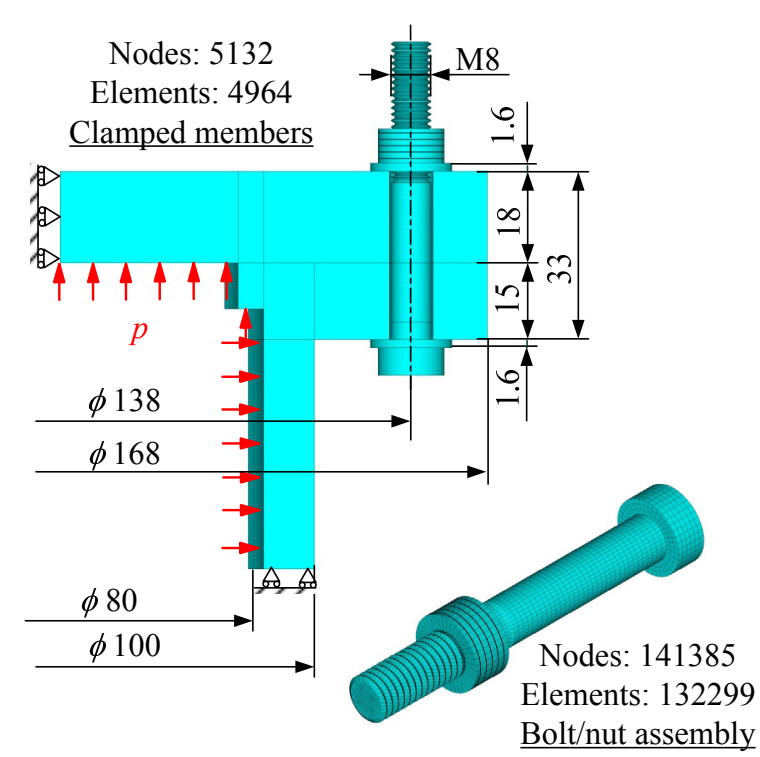

(a) With axi-symmetric bolt/nut assembly model

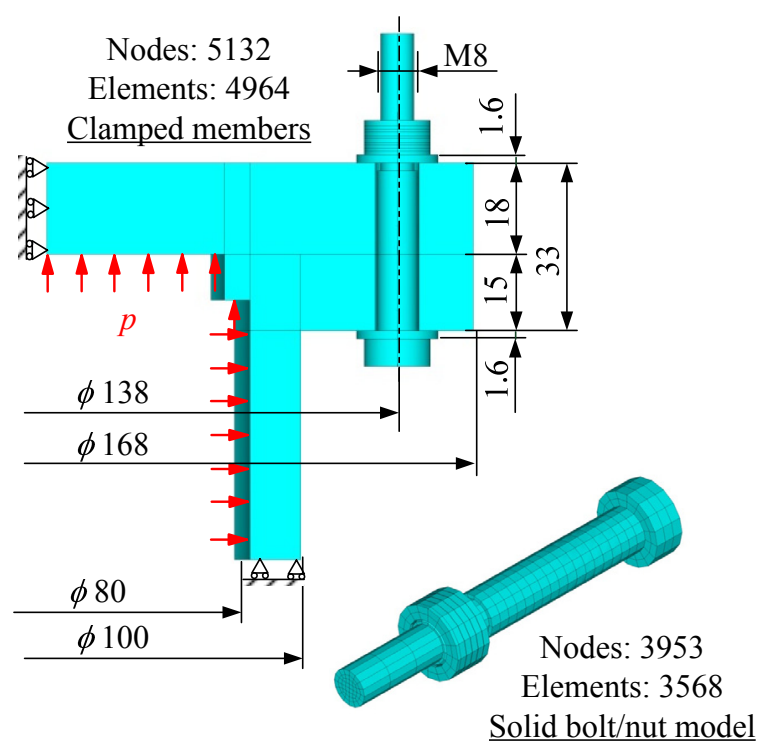

(b) With solid bolt/nut model (Model B)

Fig. 10 3D FE models (single-bolted joint model) to analyze the internal forces or the stresses acting on a bolt/nut assembly in the cylinder cover bolted joint shown in Fig. 9. The clamped members of both models consist of the same number of elements. However, the number of elements in the solid model (Model B with $\theta=60^{\circ}$ and $w=P / 16$, and $d_{\mathrm{ea}}=d_{1}$ ) is less than $1 / 30$ of those in the bolt/nut assembly model.

bolt shank. The results using the conventional model [Model A in Fig. 5 without slits $\left(C_{1}=0, C_{2}=0\right.$ and $\left.C_{3}=1\right)$ ] with cylindrical body $d_{\mathrm{eq}}=d_{1}$ are also shown for a reference. Instead of showing the stress in megapascals (MPa), the ordinates show the equivalent force $F_{\mathrm{be}}=\left(\sigma_{\mathrm{ten}} \pm \sigma_{\mathrm{ben}}\right) \cdot A_{\mathrm{b}}$, i.e., the stress multiplied by the sectional area of the bolt shank. The equivalent force at $W=0$ represents the clamp force $F$, and the middle line of maximum and minimum stresses represents the axial force $F_{\mathrm{b}}$ acting on the bolt. The difference of the bending stresses for different gauge positions 
shows the existence of the transverse force on the bolt/nut assembly. The stresses acting on the bolt obtained using the solid model [Fig. 10 (b)] coincide accurately with ones using the bolt/nut assembly model [Fig. 10 (a)], and both results show good agreement with the measurements by the strain gauges put on the bolt shank.

The conventional model seems to give also good results. However, it should be noted that the bending stress $\sigma_{\text {ben }}$ is a little bit smaller especially in the case of low clamp force $(F=6.6 \mathrm{kN})$ contrary to our expectation that higher flexural stiffness of the bolt/nut model brings higher bending stress $\sigma_{\text {ben }}$ than practical bolt/nut assembly. Such a phenomenon comes from the complexity of the equilibrium of forces and bending moment in bolted joints.

Figure 12 shows the displacements of the interface of the cover and the lower flange when the maximum force $W=9 \mathrm{kN}$ is applied to the cylinder-cover bolted joint with lowest preload $(F=6.6 \mathrm{kN})$. The amount of the separation for the conventional model is smaller than the cases with the axi-symmetric bolt/nut assembly and with Model B. This depicts the fact that the bolt/nut assembly with higher axial and flexural stiffnesses can work to prevent the separation at the contact plane (the interface between the cover and the flange), and results in the smaller slope on the bearing surfaces in this case. The effect may be dependent upon the geometry and other conditions of the bolted joint to be analyzed. Therefore, the use of bolt/nut model having the same stiffness as the practical bolt/nut assembly is essential.

Considering the fact that the solid model consisting of only about 3600 elements (almost the same as the conventional model) gives the same behavior as the bolt/nut assembly model consisting of 132 thousand elements, we can easily understand the superiority or the benefits of the solid model proposed in this study in 3D-FE analysis for bolted joints in general. It may expand the field of applications of FE analysis in the early design stage. The internal forces and the bending moment obtained using the solid model can be used as the external load on fully modeled bolt/nut assembly model with finer meshing to determine the characteristics such as the local stress acting on the bolt thread root and the trajectory of slippage between mating threads in the proceeded design stage.
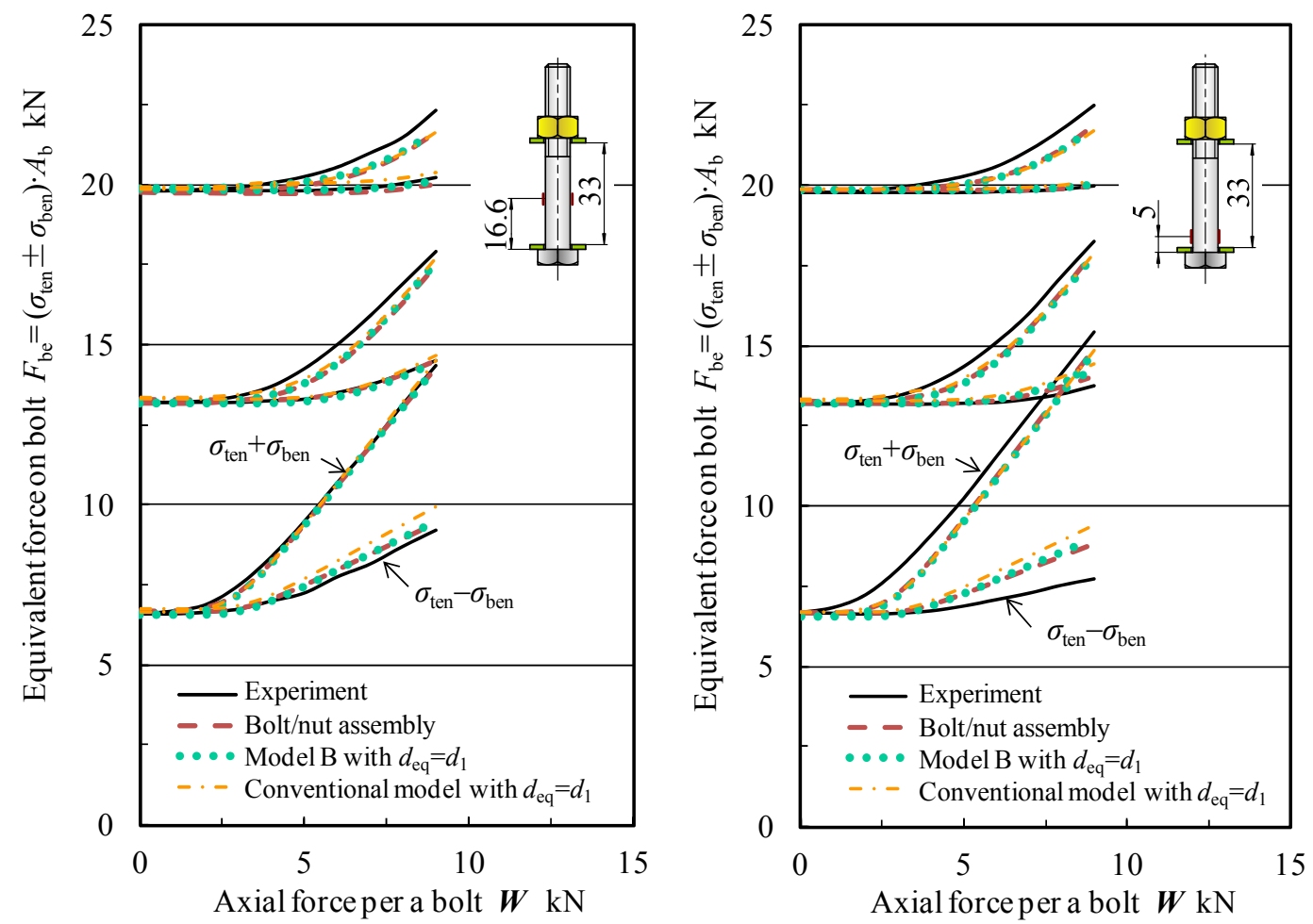

Fig. 11 Estimation of the stresses acting on the bolt/nut assembly in the cylinder cover bolted joint shown in Fig. 9. The results are obtained for three different clamp forces $(F=6.6,13.2$ and $19.8 \mathrm{kN})$ and two different gauge positions $\left(16.6 \mathrm{~mm}\right.$ and $5 \mathrm{~mm}$ from the bearing face of the bolt). The maximum stress $\left(\sigma_{\text {ten }}+\sigma_{\text {ben }}\right)$ and the minimum stress $\left(\sigma_{\text {ten }}-\sigma_{\text {ben }}\right)$ act on the inner and the outer sides of the bolt shank, respectively, see Fig. 9. The calculation by using the solid model (Model B) with only about 3600 elements coincides accurately with the one by using axi-symmetric bolt/nut assembly model with about 132 thousand elements, and both results represent the actual behavior measured by strain gauges well. The conventional model without cavities and slits $\left(C_{1}=0, C_{2}=0\right.$ and $C_{3}=1$ in Fig. 6) shown in orange dot-dash line tends to give greater axial force and smaller bending stress. 


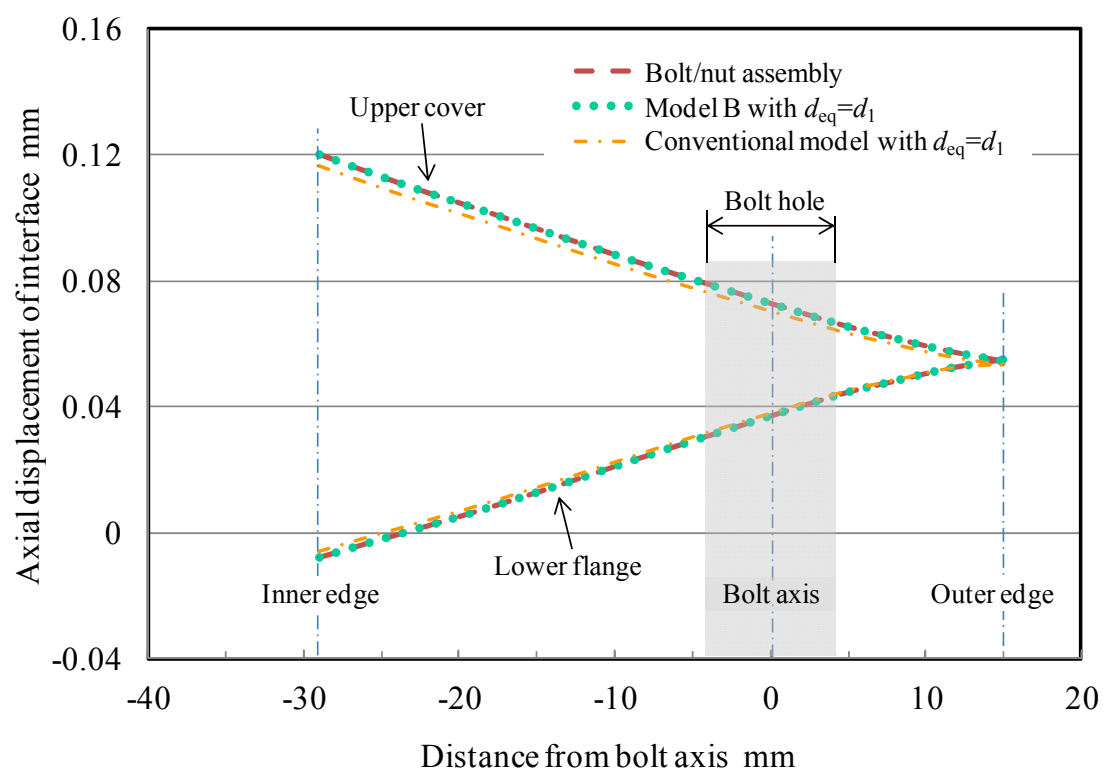

Fig. 12 Axial displacement of the interface between the cover and the lower flange $(W=9 \mathrm{kN}$ and $F=6.6 \mathrm{kN})$. The higher axial and flexural stiffnesses on the nut side of the conventional model work to prevent the separation at the contact plane (interface between the cover and the flange), and decrease the axial displacement. This leads to the lower slope on the bearing surface of nut side, and results in the lower bending moment on the conventional model of bolt/nut assembly shown in Fig. 11. The axial deformation (displacement) on the bearing surface is also smaller. However, the higher axial force is acting on the conventional model since the axial stiffness is much higher.

\section{Conclusions}

The main conclusions obtained in this study are summarized as follows:

(1) The solid model for bolt/nut assembly was developed for 3D-FE analysis of mechanical structures with bolted joints which has same axial and flexural stiffnesses as the bolt/nut assembly to be modeled.

(2) In the solid model, the free-threaded portion is transformed into the cylindrical body having equivalent diameter of $d_{1}$ (actual root diameter of thread), and the mating threads are substituted by the multiple conical cavities with pitch $=P$, inner diameter $=d_{1}$, outer diameter $=D$, the angle $\theta=60^{\circ}$ and the width $w=P / 16$. All the geometry of this solid model can be determined directly from the specifications of the practical bolt/nut assembly to be modeled.

(3) The stresses on the bolt shank in a cylinder cover bolted joint calculated by using the solid model with about only 3600 elements coincide accurately with those obtained by using the fully modeled bolt/nut assembly model with about 132 thousand elements, and show good agreement with the measurement by strain gauges as well.

(4) The solid model developed in this study can be applicable to wide varieties of bolt/nut assembly in a formulated manner, and enables to reduce drastically the total number of elements (and of contact surfaces) without downgrading the accuracy of calculation, and results in saving the preparing time for modeling and the processing time in 3D FE structural analysis for bolted joints tremendously. It may also expand the field of application of 3D-FE analysis itself in the strength design of bolted joints.

\section{Acknowledgement}

The authors wish to express their sincere thanks to Mr. Daigo Fukazawa, former student of Nagoya Institute of Technology for his contribution to the preliminary works for this study, and to Mr. Seiya Sekino, graduate student of Nagoya Institute of Technology for his additional works to verify the calculations.

\section{References}

Bickford, J. H., An Introduction to the Design and Behavior of Bolted Joints (3 ${ }^{\text {rd }}$ ed.) (1995), p. 458, Marcel Dekker 
Inc. N.Y.

Fukuoka, T., Nomura, M. and Okayama, K., Numerical analysis of load capacity and strength of rigid flanged shaft couplings, Transactions of the JSME, Vol. 80, No. 818 (2014), DOI:10.1299/transjsme.2014smm0295 (in Japanese).

Furukawa, A., Kamiya, K. and Hagiwara, M., Effect of the Residual Stress on the Fatigue Strength of a Bolt in Bolt/Nut Assemblies (Verification of the hypotheses using an axi-symmetric bolt model), Journal of Advanced Mechanical Design, Systems and Manufacturing, Vol. 6, No. 1 (2012), pp. 189-197, DOI: 10.1299/jamdsm.6.189.

Galwelat, M. and Beitz, W., Gestaltungsrichitlinien für unterschiedliche Schraubenverbindungen, Konstruktion, Vol. 33 No. 6 (1981), pp. 213-218.

Hagiwara, M. and Ikezawa, F., On the Thread Torque Induced by the Slippage at Mating Threads in Bolt/Nut Assembly under Repeated Tensile Loading, Proceedings of the $6^{\text {th }}$ International Conference on Manufacturing, Machine Design and Tribology (2015), pp. 22-23.

Hagiwara, M., Okubo, H. and Nakamura, H., Loadability of Bolt-Nut Assemblies (Effect of Nut Specifications on the Failure Modes and Failure Load), Journal of the Japan Society for Precision Engineering, Vol. 67, No. 12 (2001), pp. 1945-1949 (in Japanese).

Hagiwara, M., Sato, T. and Yoshimoto, I., Design System of Bolted Joints ( $4^{\text {th }}$ Report) - Strength Design of Cylinder-cover-bolted Joints, Journal of the Japan Society for Precision Engineering, Vol. 51, No. 4 (1985), pp. 193-198 (in Japanese).

ISO 965-1, ISO general purpose metric screw threads - Tolerances - Part 1: Principles and basic data, (1998), International Organization for Standardization.

Okubayashi, T., Hagiwara, M., Hamada M., and Hirooka, Y., Effect of Free-Threaded Portion of a Bolt on its Tensile Properties, Transactions of the Japan Society of Mechanical Engineers, Series C, Vol.72, No.718 (2006), pp. 1982-1986 (in Japanese).

VDI 2230 Blatt 1, Systematische Berechnung hochbeanspruchter Schraubenverbindungen - Zylindrische Einschraubenverbindungen (2014), p. 39, VDI-Verlag, Düsseldorf.

VDI 2230 Blatt 2, Systematische Berechnung hochbeanspruchter Schraubenverbindungen - Mehrschraubenverbindungen (2014), p. 58, VDI-Verlag, Düsseldorf. 